\title{
AVALIAÇÃO FÍSICA E ULTRASSONOGRÁFICA DA CICATRIZ EM 12 EQUINOS SUBMETIDOS À CELIOTOMIA MEDIANA COM FIO DE POLIÉSTER PARA TRATAMENTO DE CÓLICA
}

\author{
Alvaro de Paula Lage de Oliveira ${ }^{1}$ \\ Gustavo Cancian Bajotto ${ }^{2}$ \\ Odael Spadeto Junior ${ }^{3}$ \\ Giuliano Moraes Figueiró ${ }^{4}$ \\ Anderson Luiz de Araújo ${ }^{5}$ \\ Júlia da Penha Piccoli Rangel ${ }^{6}$
}

OLIVEIRA, A. de P. L. de; BAJATTO, G. C.; SPADETO JUNIOR, O.; FIGUEIRÓ, G. M.; ARAÚJO, A. L. de; RANGEL, J. da P. P. Avaliação física e ultrassonográfica da cicatriz em 12 equinos submetidos à celiotomia mediana com fio de poliéster para tratamento de cólica. Arq. Ciênc. Vet. Zool. UNIPAR, Umuarama, v. 18, n. 2, p. 121-127, abr./jun. 2015.

\begin{abstract}
RESUMO: Foi realizado um levantamento das complicações incisionais em 12 equinos submetidos à celiotomia mediana seguida de celiorrafia com fio de poliéster. Os animais foram submetidos a exame clínico e complementar. O exame clínico consistiu na inspeção e palpação da linha média. O método de exame complementar foi a ultrassonografia da linha media na área referente à cicatriz abdominal. Foram encontrados complicações incisionais em sete animais (58,3\%). As complicações incisionais encontradas foram fibrose $(41,6 \%)$, reação ao fio de sutura $(41,6 \%)$, fístulas $(16,6 \%)$, edema peri-incisional (25\%), infecção incisional (16,6\%), drenagem incisional $(16,6 \%)$. No exame indireto foi observado edema pós-operatório, pequenas áreas focais de líquido ao redor do fio de sutura. Mesmo com as complicações incisionais encontradas e o número de animais examinados, o fio de poliéster mostrou-se resistente e seguro podendo ser utilizado na laparorrafia mediana em equinos.
\end{abstract}

PALAVRAS-CHAVE: Celiorrafia mediana. Complicações incisionais. Equino. Fio de sutura.

\section{PHYSICAL AND ULTRASONOGRAPHIC ASSESSMENT OF SCAR FROM CELIORRHAPHY WITH POLYESTER THREAD IN HORSES UNDERGOING TREATMENT OF ACUTE ABDOMINAL COLIC}

\begin{abstract}
A survey on the incisional complications in twelve horses submitted to median celiotomy followed with celiorrhaphy with polyester suture was performed. The animals were submitted to clinical and complementary examination. Inspection and palpation of the midline for clinical evaluation was performed. Ultrasonography of the abdominal scar of the midline area was used as an indirect test. Seven animals presented incisional complications (58.3\%), such as fibrosis (41.6\%), suture material reaction (41.6\%), fistulas (16.6\%), peri-incisional edema (25\%), wound infection (16.6\%), and incisional drainage (16.6\%). In indirect evaluation, post-operative edema was observed, as well as small focal areas with fluid around the suture. Despite the incisional complications found and the small number of animals examined, it was concluded that polyester suture proved to be resilient and safe, and can be used in median celiorrhaphy in horses.

KEYWORDS: Celiorrhaphy median. Equine. Incisional complications. Suture filament.

\section{EVALUACIÓN FÍSICA Y ECOGRAFIA DE CICATRIZ EN 12 EQUINOS SOMETIDOS A CELIOTOMIA MEDIANA CON HILO DE POLIÉSTER PARA TRATAMIENTO DEL CÓLICO}

RESUMEN: Una encuesta sobre las complicaciones incisionales en 12 equinos sujetados a una celiotomía mediana seguida de una celiorrafia con hilo de poliéster. Los animales fueron sometidos a exámenes clínicos y complementarios. El examen clínico incluyó la inspección y palpación de la línea mediana. Otro método evaluativo fue la ecografía de la línea media en la zona referente a la cicatriz abdominal. Complicaciones incisionales fueron encontradas en siete animales $(58,3 \%)$. Las complicaciones incisionales encontradas fueron fibrosis $(41,6 \%)$, reacción al hilo de sutura (41,6\%), fístulas (16,6\%), edema perincisional (25\%), infecciones incisionales $(16,6 \%)$ y drenaje incisional $(16,6 \%)$. En el examen indirecto se ha observado edema postoperatorio, pequeñas áreas focales de líquido alrededor del hilo de sutura. A pesar de las complicaciones incisionales encontradas y el número de animales examinados, el hilo de poliéster demostró resistencia, seguridad y puede ser utilizado en celiorrafia mediana en equinos.

PALABRAS CLAVE: Celiorrafia mediana. Complicaciones incisionales. Equinos. Hilo de sutura.

DOI: https://doi.org/10.25110/arqvet.v18i2.2015.5383

${ }^{1}$ Doutorando em Ciência Animal pela Universidade Federal de Minas Gerais. Departamento de Clínica e Cirurgia Veterinárias. Avenida Presidente Antônio Carlos, 6627. Campus Pampulha da UFMG.CEP: 31.270-901. Belo horizonte-MG. alvaroploliveira@gmail.com

${ }^{2}$ Médico Veterinário autônomo.

${ }^{3}$ Docente pela Universidade Vila Velha-UVV, Doutorando em Ciência Animal pela Universidade Federal de Minas Gerais.Vila Velha-ES

${ }^{4}$ Docente pela Universidade Federal de Santa Catarina- UFSC. Curitibanos-SC.

${ }^{5}$ Técnico administrativo em educação da Universidade Federal da Bahia-UFBA, Cirurgia de grandes animais. Salvador-BA.

${ }^{6}$ Docente pela Universidade Vila Velha-UVV. Vila Velha-ES. 


\section{Introdução}

A cólica é um dos problemas mais comuns e mais desafiadores que os veterinários de equinos encontram. Embora a maioria dos casos possa ser tratada clinicamente, até 10\% necessitam de intervenção cirúrgica (DUKTI; WHITE, 2009).

Apesar dos avanços na gastroenterologia equina, uma proporção substancial destes animais não conseguem sobreviver à cirurgia de cólica e outros, mesmo operados, não conseguem retornar às atividades com o nível de desempenho esperado (PHILLIPS; WALMSLEY, 1993).

Nas últimas décadas observou-se um aumento na taxa de sobrevivência dos animais operados, havendo também um aumento na frequência de complicações pós-operatórias não fatais (INGLE-FEHR et al., 1997). De acordo com Pagliosa \& Alves (2004) e Kobluk et al. (1989) a prevalência de complicações incisionais nas celiotomias medianas pode variar de $35 \%$ a $87,5 \%$. As complicações incisionais na celiotomia mediana retardam o tempo de cicatrização da ferida cirúrgica, aumentando o período de convalescença dos animais, consequentemente se tornando um fator de risco (PAGLIOSA; ALVES,2004).

Para detecção de possíveis alterações na parede abdominal de animais submetidos àceliotomia mediana é realizado exame físico da área abdominal referente à celiorrafia por meio da inspeção e palpação. Porém, o exame físico é um método direto de avaliação, podendo muitas vezes se tornar inconclusivo, devido à presença de área extensa de edema e dor no pós-operatório, tornando difícil a avaliação da cicatriz abdominal na celiorrafia (NASCIMENTO, 2008).

Para minimizar erros provenientes do exame direto pode ser empregado o exame ultrassonográfico. É um exame mais sensível e indireto de avaliação, permitindo detectar complicações no pós-cirúrgico imediato e tardio de animais submetidos à celiotomia mediana, auxiliando assim, no diagnóstico e na tomada de decisão pelo médico veterinário (NASCIMENTO, 2008; PROTOPAPAS, 2000; HONNAS; COHEN, 1997; INGLE-FEHR et al., 1997; WILSON et al., 1995; PHILLIPS; WALMSLEY, 1993; CAMPBELL; BAILEY, 1992; GIBSON et al., 1989; KUBLOK et al., 1989; TURNER et al.,1988; McllWRAITH, 1978).

O objetivo desse trabalho foi realizar um levantamento das complicações incisionais em cicatriz de celiorrafia mediana com fio de poliéster em equinos submetidos à celiotomia mediana para o tratamento de cólica.

\section{Material e métodos}

Foram avaliados 12 equinos, cinco fêmeas e sete machos, com peso entre 353 e $510 \mathrm{~kg}$, submetidos à celiotomia mediana para tratamento de cólica, com diferentes tempos de pós-operatório,operados na região da Grande Vitória-ES no período entre abril de 2013 a outubro de 2014.

Todos os animais integrantes deste estudo foram anestesiados com o seguinte protocolo: medicação pré-anestésica com xilazina $(0,7 \mathrm{mg} / \mathrm{kg} / \mathrm{IV})$, seguido de indução com cetamina $(2,2 \mathrm{mg} / \mathrm{kg} / \mathrm{IV})$ associado a diazepam $(0,1 \mathrm{mg} / \mathrm{kg} /$ IV) e manutenção com isoflurano. A técnica cirúrgica consistiu de incisão de pele e subcutâneo seguida exposição e diérese da linha branca com bisturi e/ou tesoura metzenbaum.
Após visualização e separação da gordura retroperitoneal uma pequena incisão do peritônio era realizada com o bisturi e em seguida era ampliada com os dedos indicadores. Os tamanhos das incisões variaram entre 35 a $50 \mathrm{~cm}$ de comprimento. Após resolução das afecções entéricas a linha branca era suturada com fio de poliéster $n^{\circ} 2$ em plano de sutura simples contínuo que era interrompido na metade do comprimento da incisão. Em seguida era realizada a síntese de subcutâneo com fio de ácido poliglicólico $\mathrm{n}^{\circ} 0$ e a pele com fio de nylon $n^{0} 1$. O protocolo pós-operatório utilizado foi flunixin meglumine $(1,1 \mathrm{mg} / \mathrm{kg} / \mathrm{IV} / \mathrm{SID})$ durante cinco dias, DMSO solução à $10 \%(100 \mathrm{~mL}$ em $900 \mathrm{Ml}$ de solução fisiológica/IV/SID) durante três dias, gentamicina $(6 \mathrm{mg} / \mathrm{kg} / \mathrm{IV} /$ SID) durante três dias, metronidazol (15 mg/ $\mathrm{kg} / \mathrm{IV} / \mathrm{SID}) \mathrm{du}-$ rante três dias, penicilina benzatina $(22.000 \mathrm{UI} / \mathrm{kg} / \mathrm{IM} / \mathrm{SID})$ durante sete dias, heparina (40 UI/SC/TID) durante três dias, lidocaína bolus de $(1,33 \mathrm{mg} / \mathrm{Kg})$ seguido de infusão contínua ( $3 \mathrm{mg} / \mathrm{kg} / \mathrm{h}$ ) durante três dias de acordo com avaliação do animal,utilização de compressa quente/fria duas vezes ao dia em região peri-incisional, curativos diários até o décimo dia. Os animais foram submetidos a exame clínico e complementar. O exame clínico consistiu na inspeção e palpação da linha média. $\mathrm{O}$ exame complementar foi realizado por meio da ultrassonografia (aparelho Honda ${ }^{\circledR}$ modelo HS$101 \mathrm{~V}$ ), da linha media na área referente à cicatriz abdominal, com transdutor linear de 7,5 MHz. As imagens ultrassonográficas foram realizadas nos planos transversais e longitudinais. O objetivo dos exames foi identificar alterações como edema subcutâneo, fístulas, hérnias, reações ao fio.As imagens ultrassonográficas e os achados no exame clínico foram digitados e arquivados juntamente com fotos individuais dos animais (adaptado de NASCIMENTO,2008).

\section{Resultados e discussão}

Fatores como idade superior a um ano e peso do animal superior a $300 \mathrm{~kg}$, podem contribuir para o surgimento de complicações incisionais (HARDY; RAKESTRAW, 2006; FREEMAN, 2005; FREEMAN et al., 2002; INGLE-FEHR et al.,1997; KOBLUK et al., 1989).Isso vai ao encontro com os achados nesse levantamento, visto que os animais examinados possuíam mais de um ano de idade (média 6,1a) e média de peso $373,4 \mathrm{~kg}$ (Tabela 1).

O exame clínico (inspeção e palpação) permitiu detectar de forma satisfatória edema, fistulas com drenagem. Já o exame complementar (ultrassonográfico) possibilitou a confirmação dos achados encontrados no exame indireto e ainda a visualização da presença de líquido ao redor dos nós cirúrgicos (Tabela 2). Dessa forma é possível afirmar que esses métodos foram eficazes para verificação da presença das complicações incisionais em celiotomia mediana de equinos. Isso também é descrito por Nascimento (2008).

No exame de inspeção, apenas foi possível visualizar o fio (aparente) em um animal ( $\left.n^{\circ} 10\right)$ (Figura 1). Isso foi interpretado como uma reação de corpo estranho ao fio de poliéster. No exame de palpação nos animais número $1,2,3,4,5$ e 9 foi possível perceber a presença do fio apesar de pouco evidente, nos animais número 6,7,8,11 e 12 observou fio evidente. No exame direto (US), foi encontrado fio de sutura evidente em todos os animais (Tabela 2). 
Figura 1: Presença de fio de poliéster (seta preta) aparente ao exame de inspeção. Aumento de volume na linha de sutura e presença do fio de poliéster no animal 10 (circulo) (Arquivo pessoal).

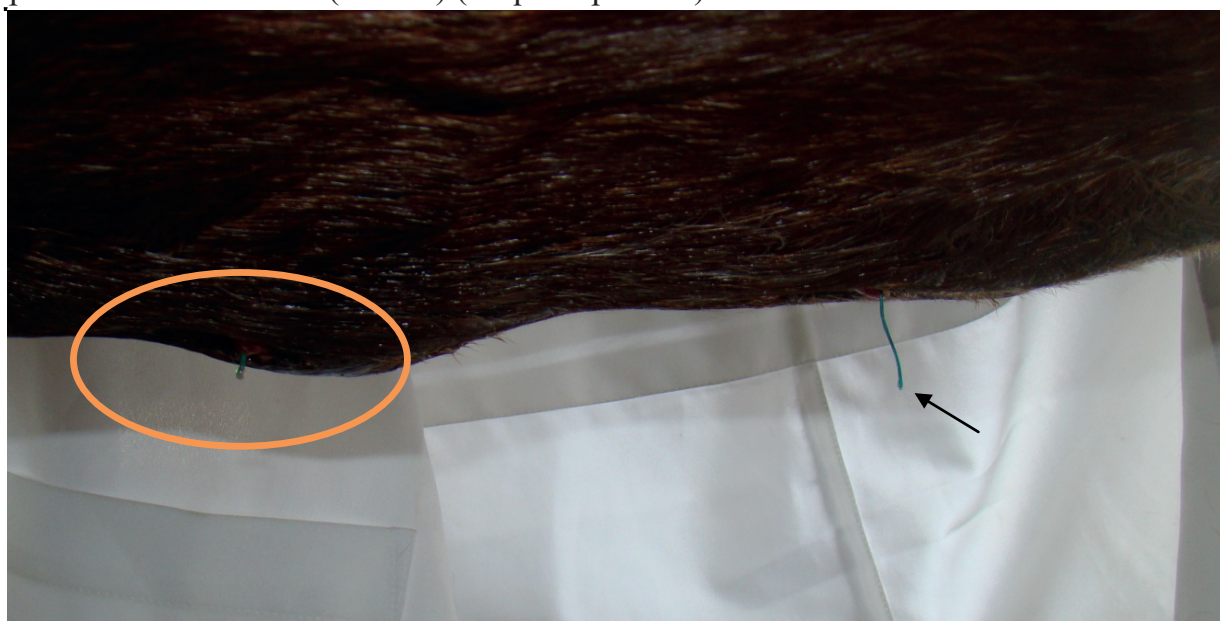

Tabela 1: Informações obtidas por meio da avaliação realizada nos 12 equinos com síndrome cólica submetidos à celiorrafia mediana utilizando fio de poliéster na rafia de linha média no HV- UVV e HV - BelaVista.

\begin{tabular}{|c|c|c|c|c|c|c|}
\hline $\mathrm{N}$ & Sexo & Raça & Idade & Peso & Patologia cirúrgica & T.O \\
\hline 1 & $\mathrm{~F}$ & MM & $9 \mathrm{a}$ & $390 \mathrm{~kg}$ & Encarceramento nefro-esplênico & $4 \mathrm{~m}$ \\
\hline 2 & M & MM & $4 a$ & $380 \mathrm{~kg}$ & Deslocamento e compactação de cólon maior & $5 \mathrm{~m}$ \\
\hline 3 & $\mathrm{~F}$ & QM & $2,6 \mathrm{a}$ & $368 \mathrm{~kg}$ & Encarceramento nefro-esplênico & $6 \mathrm{~m}$ \\
\hline 4 & $\mathrm{~F}$ & QM & $1,6 \mathrm{a}$ & $353 \mathrm{Kg}$ & Fecaloma em cólon menor & $1 \mathrm{~m}$ \\
\hline 5 & M & QM & $3 a$ & $390 \mathrm{Kg}$ & Deslocamento e compactação de cólon maior & $6 \mathrm{~m}$ \\
\hline 6 & $\mathrm{~F}$ & MM & $6 \mathrm{a}$ & $375 \mathrm{Kg}$ & Compactação de cólon maior e fecaloma no cólon menor & $1,5 \mathrm{a}$ \\
\hline $7 *$ & $\mathrm{~F}$ & Pônei & $1 \mathrm{a}$ & $70 \mathrm{~kg}$ & $\begin{array}{l}1^{\mathrm{o}}: \text { sablose e fecaloma colón menor } 2^{\mathrm{o}} \text { : compactação de cólon } \\
\text { transverso e fecaloma cólon menor }\end{array}$ & $11 \mathrm{~m} / 7 \mathrm{~m}$ \\
\hline 8 & M & QM & $15 \mathrm{a}$ & $400 \mathrm{~kg}$ & Compactação de jejuno & $14 d$ \\
\hline 9 & M & $\mathrm{C}$ & $4,7 \mathrm{a}$ & $510 \mathrm{~kg}$ & Hérnia inguinal & $3 \mathrm{~m}$ \\
\hline 10 & M & MM & $9 \mathrm{a}$ & $410 \mathrm{~kg}$ & Dilatação com aderência de jejuno & $1,6 \mathrm{a}$ \\
\hline 11 & M & QM & $3 a$ & $430 \mathrm{~kg}$ & Fecaloma de cólon menor & $2 \mathrm{~m}$ \\
\hline 12 & M & $\mathrm{PH}$ & $15 \mathrm{a}$ & $405 \mathrm{~kg}$ & Encarceramento nefro-esplênico & $10 \mathrm{~d}$ \\
\hline
\end{tabular}

MM-Margalarga Marchador; QM-Quarto de Milha; PH- Paint Horse; C-Campolina; F-Fêmea; M-Macho; T.O-Tempo de Operado; a-anos; m-meses.

*Animal submetido a mais de uma celitomia mediana para tratamento de cólica. Adaptado de Nascimento (2008).

Dos 12 animais examinados, 58,3\% desenvolveram complicações incisionais após realização da celiotomia mediana (Tabela 2). Esses dados foram superiores aos encontrados por Kobluk et al. (1989) em um estudo realizado com 70 equinos onde obtiveram $28,4 \%$ de alterações incisionais e do levantamento realizado por Wilson et al. (1995) que verificaram complicações incisionais em 274 animais e obteve taxa de incidência de $40 \%$ após a primeira celiotomia. Já Honnas e Cohen (1997) relatam que a taxa de complicações encontradas em 210 animais avaliados foi de $76,7 \%$, resultado maior que o encontrado nessa avaliação.

Em um estudo realizado com 12 animais Kobluk et al. (1989) relatam que utilizando como fio de sutura o poliéster na celiorrafia mediana, obteve uma taxa de 33,3\% de alterações incisionais. Comparando ao resultado encontrado no presente pesquisa, a taxa encontrada $(58,3 \%)$ foi maior do que a descrita pelo autor.O resultado encontrado pode ter ocorrido devido às características físicas do fio de poliéster devido este ser multifilamentar e permitir a ocorrência de complicações incisionais (FOSSUM, 2005; RIBEIRO et al.,
2005; PAGLIOSA; ALVES, 2004; FREEMAN et al., 2002; CAMPBELL; BAILEY, 1992).

Wilson et al. (1995) relatam que a taxa de prevalência na ocorrência das complicações incisionais em animais com idade superior a um anofoi de $43 \%$. Essa mesma taxa foi observada em animais com peso superior a $300 \mathrm{~kg}$. Todos os animais com complicação incisionais examinados nesse levantamento apresentaram idade acima de um ano (média 6,1 anos)e seis animais tiveram peso superior a $300 \mathrm{~kg}$ (média $373,4 \mathrm{~kg})$.

Ainda, Wilson et al. (1995) relatam ocorrência de complicações em $25 \%$ de machos e $30 \%$ em fêmeas. No presente levantamento, a taxa de machos $(71,4 \%)$ com alterações incisionais foi maior em comparação ao descrito por este autor. A taxa de fêmeas acometidas foi de $28,6 \%$. Tais resultados podem ser explicados pela maior criação intensiva de machos. Esse fator pode predispor a casos de cólica com evolução cirúrgica.

A realização de uma segunda cirurgia de celiotomia mediana pode favorecer o surgimento de complicações pós- 
-operatórias (HARDY; RAKESTRAW, 2006; FREEMAN, 2005; INGLE-FEHR et al.,1997; KOBLUK et al., 1989). Em um estudo realizado por Kobluk et al. (1989) em cinco animais submetidos a mais de um procedimento cirúrgico, a taxa encontrada de alterações foi de $100 \%$. Segundo Honnas e Cohen (1997) em uma pesquisa realizada com 161 animais, $100 \%$ apresentaram complicação incisional após realização de múltiplas cirurgias. Dos animais examinados, apenas um animal $\left(n^{\circ} 7\right)$, foi submetido a mais de uma celiotomia mediana. Este animal apresentou áreas de fibrose localizada em terço cranial, médio e caudal da ferida cirúrgica, provavelmente nos locais dos nós cirúrgicos.Nas duas intervenções a que este animal foi submetido foram utilizadas fio de poliéster na celiorrafia (Figura 2).

Figura 2: Cicatriz abdominal referente ao procedimento cirúrgico de celiotomia mediana anteriormente realizada no animal $\mathrm{n}^{\circ} 7$ (A). Segunda intervenção cirúrgica abdominal quatro meses após o primeiro procedimento, nota-se a presença do fio de poliéster preservado em linha média (seta) (B) (Arquivo pessoal).

Segundo Nascimento (2008) num estudo realizado com 16 animais, todos portadores de complicações incisionais, as patologias cirúrgicas intestinais mais observadas foram às relacionadas ao cólon maior. No presente estudo as patologias cirúrgicas mais observadas em animais com alteração na cicatriz também foram em cólon maior (2 animais com encarceramento nefro-esplênico) assim como o descrito por esse autor.

Tabela 2: Resultados dos exames clínicos e US realizados no período de 02 de Outubro a 15 de Outubro de 2014 nos 12 equinos com síndrome cólica submetidos à celiotomia mediana (com diferentes tempos de pós-cirúrgico) utilizando fio de poliéster na celiorrafia de linha média para o tratamento de cólica na HV- UVV e HV- Bela Vista.

\begin{tabular}{ccccc}
\hline $\mathbf{N}$ & Inspeção & Palpação & US & Atividade desenvolvida \\
\hline 1 & Ndn & F.P.E & A.L.Dc/F.E & C.M \\
2 & Ndn & F.P.E & A.V.Dc/F.E & C.M \\
3 & A.V.Di.E & F/F.P.E & F/R.F/F.E & $\mathrm{Cr}$ \\
4 & Ndn & F.P.E & F.E & $\mathrm{Cr}$ \\
5 & Ndn & F.P.E & A.L.Dc/F.E & P.T \\
6 & Ndn & F.E & A.L.Dc/F.E & C.M \\
7 & A.V.E & F/F.E & F/F.E & E \\
8 & Fist/A.V.Di.E/S/I.I & S/Fist/F.E/A.V.P.I/I.I & Fist/R.F/A.V.P.I/F.E/F.P/E.P.I & P.L \\
9 & A.V.Di.E & F/F.P.E & F/R.F/F.P/F.E & Ca \\
10 & A.V.Di.E/F.A/D.P/I.I & Fist/A.V.P.I/F.E/F.A/I.I & R.F/Fist/E.P.A/F/E.P.I & $\mathrm{R}$ \\
11 & Ndn & F.Di/E.P.A/F.E & F/E.P.A/F.E & Cr \\
12 & A.V.P.I & A.V.P.I/F.E & E.P.A/F.E/R.F/E.P.I & V
\end{tabular}

Ndn - Nada digno de nota; Físt. - Fístula; S - Seroma; I.I - Infecção incisional; A.V.Di.E. - Aumento de volume difuso evidente; A.V.Dc. - Aumento de volume discreto; A.V.P.I - Aumento de volume peri-incisional; A.L.Dc. - Acúmulo de líquido discreto; A.V.E - Aumento de volume evidente; F.P.E. - Fio pouco evidente; F.E. - Fio evidente; F.A - Fio aparente; F - Fibrose; F.Di - Fibrose discreta; RF- Reação ao fio; F.P - Falha em parede abdominal; E.P.A - Espessamento de parede abdominal; E.P.I- Edema peri-incisional C.M - Concurso de marcha; Cr - Criação; P.T - Prova de tambor; P.L - Prova de laço; E - exposição; Ca- Cavalgada; R- Reprodução; V- Vaquejada; adaptado de NASCIMENTO (2008).

O fio de poliéster é considerado um fio mais resistente em relação aos demais fios utilizados na celiorrafia mediana, além de permitir maior segurança no nó da sutura
(TROSTLE et al., 1994; CAMPBELL; BAILEY, 1992). Entretanto, um estudo realizado por Turner et al. (1988), que avaliaram o grau de influência do material de sutura no surgi- 
mento das hérnias incisionais, mostrou a incidência de 5,7\% e $8,3 \%$ com uso de fio de sutura de poliglactina 910 e poliéster respectivamente. No presente estudo não foi encontrado nenhum animal com deiscência de sutura e hérnia incisional concordando com Campbell e Bailey(1992) e discordando de Turner et al. (1988).

Dos animais (Figura 3) examinados dois $(16,6 \%)$ desenvolveram infecção incisional, drenagem incisional e fístulas. Fossum (2005), Ribeiro et al. (2005), Pagliosa e Al- ves (2004), Freeman et al. (2002), Campbell e Bailey(1992) relatam que fios multifilamentares podem favorecer o aparecimento de infecções e consequentemente formação de fístulas com drenagem, devido a capilaridade existente nesses tipos de fios. Isso justificaria os achados encontrados.GIBSON et al. (1989) relatam ainda a formação de fístula devido ao uso de material de sutura não absorvível, característica física do fio de poliéster.

Figura 3: Achados ultrassonográficos abdominais. Animais 8 e 10 respectivamente (A,B), ambos com presença de área hipoecóica (circulo) sugestivo de trajeto fistuloso na região do ponto de drenagem visualizado à inspeção (Arquivo pessoal).

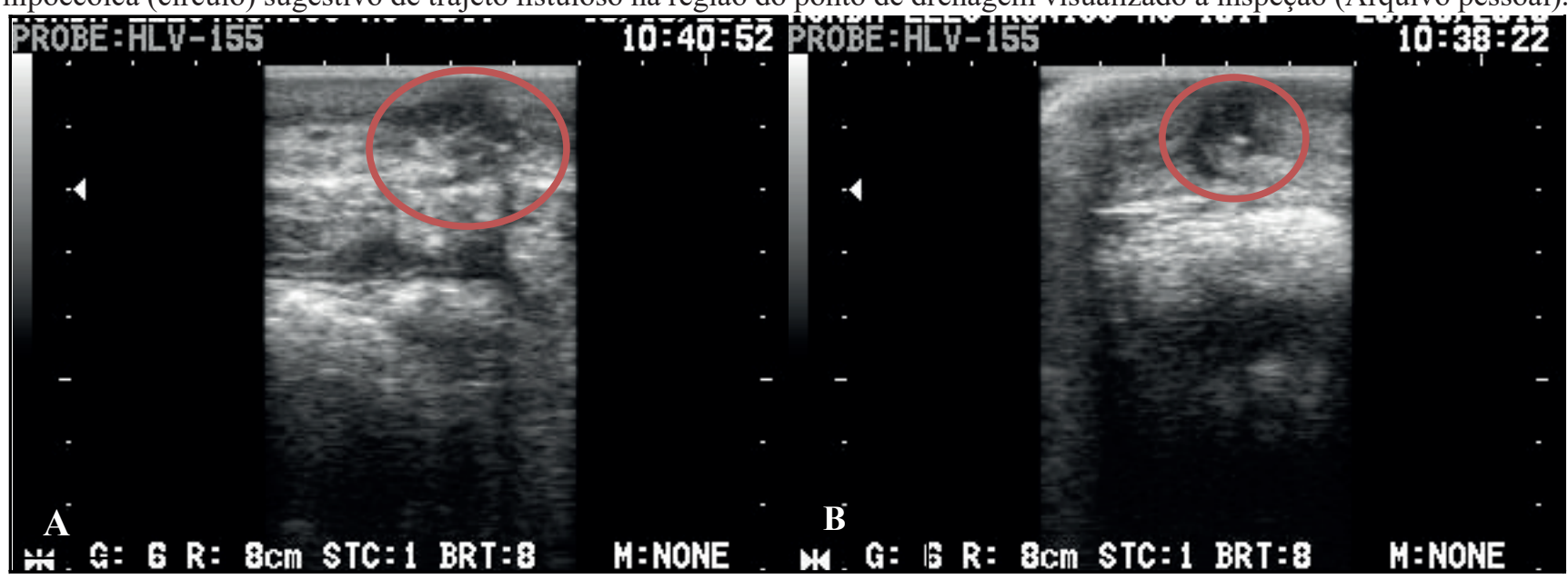

Um dos animais, mesmo passado 14 dias de pós-operatório, permanecia sob tratamentos diários com curativos da ferida incisional para controle de infecção. Além das particularidades já mencionadas do fio, Ingle-Fehr et al. (1997) mencionama presença de microrganismos patogênicos no ambiente hospitalar, na equipe cirúrgica e nos materiais que entram em contato com a ferida cirúrgica, esses seriam outros fatoresque justificariam esses achados.

Figura 4: Achado ultrassonográfico abdominal no animal cinco. Presença discreta área hipoecóica (círculo) sugerindo discreto acúmulo líquido ao redor do fio de poliéster (Arquivo pessoal).

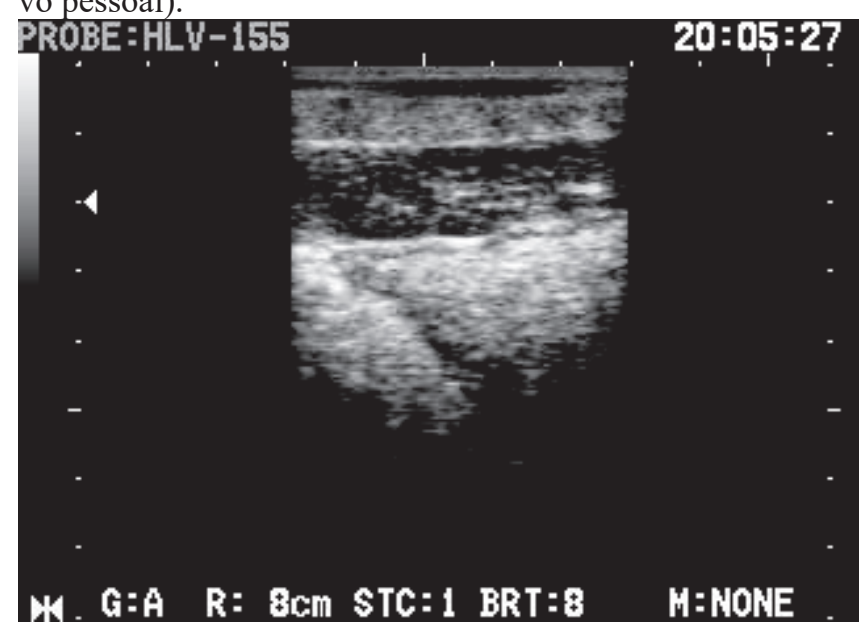

A fibrose foi encontrada em cinco animais $\left(\mathrm{n}^{\circ}\right.$ 3,7,9,10 e 11) (Figura 5).Esse achado é uma exacerbação da reação cicatricial, provavelmente, provocada pelo fio de poliéster. O tecido fibroso fornece sustentação à linha de cicatriz,
O discreto acúmulo de líquido ao redor do fio observado por meio dos exames ultrassonográficos (Figura 4) em quatro animais pode indicar o início de uma reação ao próprio fio. Freeman et al. (2002)descreve que outro fator que contribuiria para a ocorrência desse acúmulo de líquido seria o tempo de pós-operatório relativamente curto. Os três cavalos ( $n^{\circ} 1,5$ e 6$)$ possuíam menos de seis meses de operados.

mas pode trazer prejuízos estéticos. Porém a tendência deste tecido fibroso é que remodele a partir dos doze meses e se torne menos perceptível (FISCHER, 2006). De acordo com Nigro et al. (1993) a fibrose formada ao redor do fio de sutura e por todo seguimento da ferida cicatricial é causada pela reação ao fio de sutura.

Figura 5: Presença de aumento de volume em região de linha média abdominal (exacerbação da reação cicatricial) com característica de fibrose à palpação no animal número 9, três meses após realização de celiotomia mediana. Foto com vista ventral (A) e lateral (B) do abdômen (Arquivo pessoal).

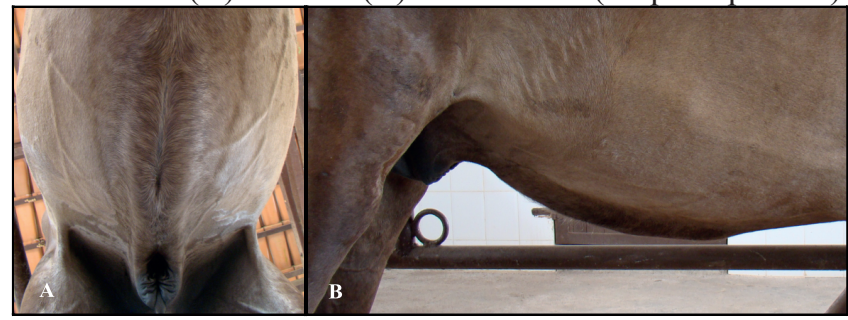

A taxa de incidência descrita na ocorrência de fístulas por Kobluk et al. (1989) que examinaram doze cavalos submetidos à celiotomia seguida de celiorrafia com fio de 
poliésterfoi de $8,3 \%$, diferente do encontrado nesse levantamento $(16,6 \%)$. Esse autor afirma que um dos principais problemas com o uso do fio de poliéster é a formação de fístulas.

Segundo Nascimento (2008) em um estudo com 16 animais, 18,7\% desenvolveram fístula quando usado fio de sutura de poliamida (nylon) na celiorrafia mediana. Os achados relatados por esse autor foram superiores ao encontrado nesse levantamento $(16,6 \%)$.

De acordo com Honnas e Cohen (1997) no estudo realizado com 210 animais, a taxa de incidência de infecção incisional encontrada foi $25,4 \%$. No presente estudo dois animais ( $n^{\circ} 8$ e $\left.n^{\circ} 10\right)$ apresentaram infecção incisional(16,6\%). Resultado menor quando comparado com estudo realizado por este autor.

Wilson et al. (1995) relatam num estudo realizado com 274 equinos submetidos à celiotomia mediana, drenagem incisional presente em $36 \%$ dos animais examinados. Nascimento (2008) afirma em um estudo realizado com 16 animais, foi encontrada taxa de $12,5 \%$ dessa complicação utilizando fio de poliamida. No presente estudo, essa complicação foi encontrada em dois animais (16,6\%). A diferença encontrada em comparação ao estudo realizado por Wilson et al. (1995) pode ser justificada pelo número de animais examinados nesse levantamento ser menor do que os examinados por esse autor. $\mathrm{O}$ resultado encontrado nesse levantamento foi menor do que o descrito por Nascimento (2008).

De acordo com Freeman et al. (2002), edema peri-incisional é comumente observado em animais com período de pós-operatório que varia entre 5-7 dias, até semanas. Isso é justificado pela recente manipulação, trauma causado pela cirurgia e resposta inflamatória. Este fato pode justificar o edema peri-incisional encontrado nos animais 8, 10 e 12 $(25 \%)$. Os animais 8 e12 foram examinados no $14^{\circ}$ e no $10^{\circ}$ dias de pós-cirúrgico respectivamente.

White (1996), Wilson et al. (1995), Phillips e Walmsley (1993), Kobluk et al. (1989) e Gibson et al. (1989) descrevem hérnia como a complicação incisional mais comum em equinos, porém neste relato nenhum animal apresentou esta alteração.

\section{Conclusão}

A celiorrafia mediana com fio de poliéster demonstrou ser resistente e segura no presente estudo, atribuído às propriedades físicas que este fio possui, tornando a sutura mais resistente e apresentando maior estabilidade dos nós. Entretanto, devido à sua característica multifilamentar, pode haver ocorrência de infecção incisional tornando-se uma potencial fonte de formação de fístulas. Nenhuma das complicações incisionais encontradas neste estudo comprometeu o bem estar e a vida dos animais examinados, o que torna encorajador o uso deste fio na rotina cirúrgica de equinos, porém se faz necessário a realização de novos estudos com maior número de animais.

\section{Referências}

CAMPBELL, E. J.; BAILEY, J. V. Mechanical properties of suture materials in vitro and after in vivo implantation in horses. Veterinary Surgery, v. 21, n. 5, p. 355-361, 1992.
DUKTI, S.; WHITE, N. Surgical complications of colic surgery. Veterinary Clinical Equine, v. 24, n. 3, p. 515 534,2009

FISCHER, A. T. J. Colic: diagnosis, preoperative management, and surgical approachs. In: AUER, J. A.; STICK, J. A. Equine surgery. Missouri: Saunders, 2006. p. 387-394.

FOSSUM, T. W. Biomateriais, sutura e hemostasia. In: FOSSUM, T. W. et al. Cirurgia de pequenos animais. São Paulo: Roca, 2005. p. 45-60.

FREEMAN, D. E.; KÖTTING, A. K.; INOUE, O. J Abdominal closure and complications. Clinical Techniques in Equine Practice, v. 1, n 3, p. 174-187, 2002.

FREEMAN, D. E. Survival and the cost of our mistakes. American Association of Equine Practitioners. Proceedings of the AAEP, - Québec, 2005.

GIBSON, K. T. et al. Incisional hernias in the horse: incidence and predisposing factors. Veterinary Surgery, v.18, n. 5, p. 360-366, 1989.

HARDY, J.; RAKESTRAW, P. C. Postoperative care and complications associated with abdominal surgery. In: AUER, J. A.; STICK, J. A. Equine surgery. Missouri: Saunders, 2006. p. 499-515.

HONNAS, C. M.; COHEN, N. D. Risk factors for woundinfection following celiotomy in horses. Journal of the American Veterinary Medical Association, v. 210, n. 1, p. 78-81, 1997.

INGLE-FEHR, J. E. et al. Bacterial culturing of ventral median celiotomies for prediction of incisional complications in horses. Veterinary Surgery, v. 26, n. 1, p. 7-13, 1997.

KOBLUK, C. N. et al. Factors affecting incisional complication rates associated with colic surgery in horses: 78 cases (1983-1985). Journal of the American Veterinary Medical Association, v. 195, n. 5, p. 639-642, 1989.

McILWRAITH, C.W. Complications of laparotomy incisions in the horse. Proceedings of the Annual Convention of the American Association of Equine Practitioners, v. 24, p. 209-218, 1978.

NASCIMENTO, A. V. Z. Avaliação física e ultrassonográfica da cicatriz em equinos submetidos à celiotomia mediana para tratamento de cólica. 2008. 15 f. Monografia (Especialização) - Universidade Federal de Minas Gerais, belo Horizonte, 2008.

NIGRO, A. J. T. et al. Estudo comparativo, em cães, das gastrorrafias feitas com fios de polipropileno e ácido poliglicólico. Acta Cirúrgica Brasileira, v. 8, n. 3, p. 118123,1993 
PAGLIOSA, G. M.; ALVES, G. E. S. Fatores pré-

disponentes das complicações incisionais de celiotomias medianas em equinos. Ciência Rural, v. 34, p. 1655-1659, 2004.

PHILLIPS, T. J.; WALMSLEY, J. P. Retrospective analysis of the results of 151 exploratory laparotomies in horses with gastrointestinal disease. Equine Veterinary Journal, v. 25, n. 5, p. 427-431, 1993.

PROTOPAPAS, K. et al. Ultrasonographic assessment and factores associated with incisional infection and dehiscence following celiotomy in the horse. Veterinary Surgery, v. 29, p. 289, 2000.

RIBEIRO, C. M. B. Estudo clínico e histopatológico da reação tecidual às suturas interna e externa dos fios monofilamentares de nylon e poliglecaprone 25 em ratos. Acta Cirúrgica Brasileira, v. 20, n. 4, p. 284-291, 2005.

TROSTLE, S. S. et al.A study of the biomechanical properties of the adult equine linea alba: relationship of tissue bite size and suture material to breaking strength. Veterinary Surgery, v. 23, n. 6, p. 435-441, 1994.

TURNER, A. S.; YOVICH, J. V. Continuous absorbable suture pattern in the ventral midline abdominal incisions in horses. Equine Veterinary Journal, v. 20, n. 6, p. 401-405, 1988.

TURNER, A. S. Local and systemic factors affecting wound healing. Proceedings of the Annual Convention of the American Association of Equine Practitioners, v. 24, p. $355-362,1978$.

WHITE, N. A. Incisional hernia after abdominal surgery in the horse. Equine Veterinary Education, v. 8, n. 6, p. 308-312, 1996.

WHITE, N. A. Intensive care, monitoring, and complications of acute abdominal diasese. In: Equine acute abdome. Philadelphia: Lea \& Febiger, 1990. p. $310-335$.

WILSON, D. A.; BAKER, G. J.; BOERO, M. J.

Complications of celiotomy incisions in horses. Veterinary Surgery, v. 24, n. 6, p. 506-514, 1995. 